УДК 539.3

\title{
НЕЛИНЕЙНАЯ ДИНАМИКА ТОПОЛОГИЧЕСКИ ОПТИМАЛЬНОЙ НАНО БАЛКИ ТИМОШЕНКО НА ОСНОВЕ МОДИФИЦИРОВАННОЙ МОМЕНТНОЙ ТЕОРИИ
}

\author{
Павлов Сергей Петрович1', \\ zhigalovm@yandex.ru
}

Жигалов Максим Викторович1, zhigalovm@yandex.ru

\section{Захарова Алёна Александровна², zaa@tu-bryansk.ru}

\author{
Крысько Вадим Анатольевич1, \\ tak@san.ru \\ 1 Саратовский государственный технический университет им. Гагарина Ю.А., \\ Россия, 410054, г. Саратов, ул. Политехническая, 77. \\ 2 Брянский государственный технический университет, \\ Россия, 241035, Россия, г. Брянск, бул. 50 лет Октября, 7.
}

\begin{abstract}
Актуальность исследования. Для контроля данных технических операций бурения скважин, различного вида ремонта скважин, геологоразведочного бурения в нефртяной и газовой промышленности на буровых и ремонтных установках всех типов используются технологические датчики (датчики нагрузки, давления, температуры жидкости, выхода раствора, плотности жидкости). Современные датчики имеют малые размеры и для повышения чувствительности изготавливаются на базе наноэлектромеханических систем, которые включают в себя составляющие элементы, нано балки и нанопластины. Эти элементы работают при высоких температурах и подвергаются механическим нагрузкам различного вида. Увеличение прочности этих элементов является несомненно актуальной задачей.

Цель: построить математическую модель на базе кинематической гипотезы Тимошенко и модифицированной моментной теории чувствительного элемента в виде балки, нано электромеханического датчика под действием механических и тепловых полей; создать методологию получения оптимальной топологии нано балки для произвольной статической и динамической нагрузки и различных граничных условий с целью увеличения ее жесткости; провести сравнительный анализ статики и нелинейной динамики оптимальной и неоптимальной балок.

объекты: элемент наноэлектромеханических систем в виде балки с учетом оптимальной микроструктуры.

Методы: методы топологической оптимизации, вариационные методы, метод конечных разностей второго порядка, методы типа Рунге-Кутта, Фурье и вейвлет анализ, фазовый портрет и сечение Пуанкаре.

Результаты. Построена методология получения оптимальной микроструктуры нано балки на основе топологической оптимизации. На основе принципа Гамильтона-Остроградского построена математическая модель неоднородной в двух направлениях (по толщине и длине) нано балки Тимошенко на базе модифицированной моментной теории. Проведен сравнительньй анализ статического изгиба и нелинейной динамики оптимальной и неоптимальной нано балок.
\end{abstract}

\begin{abstract}
Ключевые слова:
Нано балка Тимошенко, наноэлектромеханические системы, температурное и силовое поля, модифицированная моментная теория упругости, метод конечных разностей, метод типа Рунге-Кутта, топологическая оптимизация, нелинейная динамика.
\end{abstract}

\section{Введение}

Современные технологические датчики, используемые при бурении скважин, должны обладать определенным набором свойств, таких как: малая масса, устойчивость к воздействию внешних силовых, электрических и тепловых нагрузок. В связи с эти возникает проблема проектирования элементов датчиков из материалов, свойства которых не зависят от температуры, которые могут работать при больших температурах (более $\pm 150{ }^{\circ} \mathrm{C}$ ), постоянных и переменных нагрузках и т. п. Одним из направлений улучшение рабочих характеристик датчиков является использование для изготовления чувствительного элемента датчика конструктивно неоднородных материалов (КНМ). КНМ представляют собой многофазные композиты с объемной долей фаз, меняющейся в любом желаемом направлении. Это дает возможность новому материалу иметь лучшие прочностные характеристики без возникновения нежелательной концентрации напряжений. Благодаря этим особенностям балки из КНМ широко используется в различных инженерных конструкциях, таких как: газовые турбины, ветровые турбины, лопасти роторов вертолетов, гребные винты кораблей, космические и морские структуры [1].

До настоящего времени закон изменения упругих свойств, то есть микроструктура, таких элементов задавалась заранее конструктором. Изменение свойств материала вдоль произвольного направления выражалась степенным или экспоненциальным законами, которые являются самыми известными из законов для описания изменения свойств КНМ [2-4].

Топологическая оптимизация механических структур становится распространенным инструментом при 
проектировании структур с улучшенными физическими характеристиками. Для проектирования микроструктур используются такие методы, как: метод рациональной аппроксимации материала RAMP (rational approximation of material properties) [5], метод SIMP (solid isotropic material with penalization) [6], метод эволюционной структурной оптимизации (ESO) [7] и метод фиксации уровня (level set method) [8]. Среди данных методов использование RAMP приобретает все большую популярность для задач с температурными воздействиями. Основная идея этого метода заключается в определении параметров элемента как проектных переменных, а для расчета параметров в задаче оптимизации устанавливается связь между локальными параметрами (например, плотностью) и глобальными физическими свойствами материала (например, модулем Юнга и/или коэффициентом теплопроводности). С помощью различных алгоритмов поиска оптимума материалы в области оптимизации перераспределяются таким образом, чтобы достигались желаемые функциональные характеристики конструкции. Следует отметить, что существуют два подхода к включению термических и механических критериев в задачи оптимизации топологии. Первый класс рассматривает материал-кандидат как термоупругий без полной связи как теплового, так и механического полей в топологическом смысле, где обычно задано температурное поле, а термическая нагрузка преобразуется в механическую нагрузку $[9,10]$. В таком случае конструкция, прежде всего, управляется механическими характеристиками. Второй класс рассматривает материал с учетом и тепловых, и механических полей. Задача заключается в том, чтобы сначала вычислить температурное поле, а затем соединить его с полем деформаций. В таких случаях проектирование может быть обусловлено как механической функцией цели [9], так и сопряженными механическими и тепловыми функциями [11] одновременно.

При моделировании элементов НЭМС необходимо учитывать зависимость упругих свойств конструкции от размера. В классической теории упругости не предусмотрен механизм учета размерно-зависимого поведения. Зависимость упругого поведения от размера может быть объяснена с использованием моделирования на основе молекулярной динамики (MD) или механики сплошной среды более высокого порядка. Хотя подход молекулярной динамики может обеспечить более точные приближения к реальным объектам, он слишком дорог с вычислительной точки зрения. Поэтому при моделировании мелкомасштабных структур интенсивней использовались подходы уточненных теорий упругости твердого тела. На настоящий момент известны следующие теории: градиентные теории, теория микроконтинуума и нелокальная теория. Градиентная теория упругости [12] описывает эффект зависимости упругих свойств от размера с помощью трех параметров, связанных с градиентами дилатации, девиатора тензора растяжения и тензора вращения. Теория микроконтинуума была разработана Эрингеном. Она состоит из микро- полярной теории, теории микронапряжений и микроморфной теории. Микрополярная теория [13] является самой простой среди этих теорий, в то время как микроморфная теория является наиболее общей. Более подробную информацию об этих теориях, а также их приложениях можно найти в $[14,15]$.

В нелокальной теории упругости напряжение в контрольной точке в континууме зависит от деформаций во всех точках тела, и, таким образом, размерный эффект учитывается с помощью разрешающих уравнений с использованием нелокального параметра. Нелокальная теория упругости изначально была сформулирована в интегральной форме и позже переформулирована в дифференциальной форме, которая рассматривает определенную функцию ядра интегрального уравнения. По сравнению с интегральной моделью дифференциальная широко используется для нано структур благодаря своей простоте. Кроме того, недавно был предложен еще один класс теории высших порядков, который называется нелокальной теорией градиента деформаций, основанный на сочетании нелокальной теории упругости и теории градиента деформации. В работах $[16,17]$ более подробно описана эта теория. В работе [18] с помощью нелокальной теории рассмотрен подход к построению математических моделей термомеханических процессов в деформируемом теле с учетом эффектов временной и пространственной нелокальности сплошной среды.

В модифицированной моментной теории упругости (modified couple stress theory) за счет введенного дополнительного уравнении равновесия, приводящего к симметричности тензора моментов, присутствует только один размерный параметр длины материала, отвечающий за учет размерно-зависимого поведения [19]. Это изменение привело к большой популярности этой теории в научном мире. Модифицированная моментная теория применена для исследования нелинейной динамики чувствительного элемента НЭМС в работе [20]. В данной работе для построения математической модели нано балки была выбрана модифицированная моментная теория в связи с адекватным описанием размерно-зависимого поведения и незначительным изменением известных дифференциальных уравнений полноразмерной модели.

Модель Тимошенко [21], в отличие от модели Эйлера-Бернулли, учитывает деформацию сдвига и обладает большими возможностями для изучения поведения конструкций. В [22] рассмотрены КНМ нано балки с изменением свойств по толщине материала с нелинейностью типа Кармана. Для учета размернозависимого поведения использована модифицированная моментная теория. Выведены нелинейные уравнения движения для теории балок Тимошенко. Приведено исследование влияния скалярного параметра длины, закона изменения свойств балки по толщине, деформации сдвига и геометрической нелинейности на статические прогибы балки. Однако в этих работах не учитывалось смещение линии изгиба, что приводило к существенно более сложным уравнениям. В известной авторам литературе отсутствуют работы, посвященные исследованию моделей балок из мате- 
риалов с переменными в двух направлениях (по толщине и длине) свойствами.

Как показывает обзор литературы, до настоящего времени микроструктура балочных элементов определялась без использования методов оптимизации, а задавалась конструктором.

В данной работе построена многошаговая методология оптимизации армирования балки с заданным материалом с целью увеличения ее сопротивления действующим нагрузкам. Идея методологии: на первом этапе для конкретных условий нагружения, начальных и граничных условий балки, находящейся в температурном поле, проводится топологическая оптимизация структуры армирования балки заданным композитом по критерию минимума податливости (максимума жесткости). В каждом из этих случаев оптимальная структура армирования является оригинальной для данных условий нагружения и закрепления. В результате балка с оптимальной микроструктурой приобретает неоднородность в двух направлениях: по толщине и по длине.

На втором этапе исследуется статическое и динамическое поведение балки, математическая модель которой построена на базе гипотез Тимошенко, модифицированной моментной теории с учетом геометрической нелинейности по Карману. Учет влияния температурного поля вводится на основе соотношений Дюамеля-Неймана. Температурное поле не задается произвольным образом, а определяется из решения двумерного уравнения теплопроводности как стационарного, так и нестационарного.

\section{Постановка задачи}

Формулировка методологии топологической оптимизации (1 этап)

Рассмотрим двумерную упругую область $\Omega=\left\{x \in[0, a] ;-\frac{h}{2} \leq z \leq \frac{h}{2}\right\}$, ограниченную замкнутой поверхностью $\Gamma=\Gamma_{1} \cup \Gamma_{2} \cup \Gamma_{3}$, находящуюся в плоском напряженном состоянии (рис. 1). На $\Gamma_{1}$ задано граничное условие различного вида, $\Gamma_{2}$ не закреплена и не нагружена. На границе $\Gamma_{3}$ действует вертикальная нагрузка $F$, направленная вниз. Предполагается, что материал линейно упругий и изотропный.

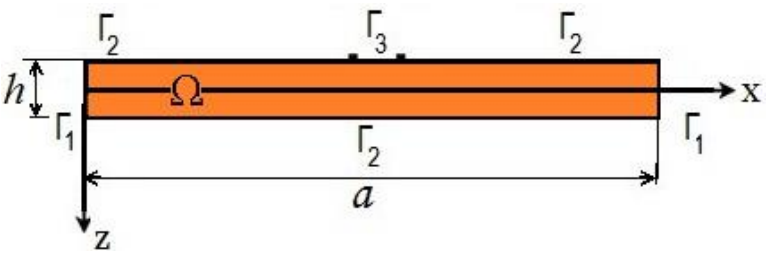

Рис. 1. Расчетная модель

Fig. 1. Calculation model

Область находится в постоянном температурном поле $T(\mathbf{x}), \mathbf{x}=\{x, z\}$. Обозначим $\theta=T(\mathbf{x})-T_{0}-$ изменение температуры относительно начальной температуры $T_{0}$, где $T_{0}$ - температура в исходном состоянии.

Для поля смещений $\left(u_{1}, u_{3}\right)$ уравнение равновесия имеют вид

$$
\sigma_{i j, j}=0 \text { в } \Omega,
$$

где $\sigma_{i j}-$ тензор напряжений. Связь линейных деформаций с перемещениями определяется по соотношениям

$$
\varepsilon_{i j}=\frac{1}{2}\left(u_{i, j}+u_{j, i}\right), i, j=1,2 .
$$

Соотношение напряжение-деформация записывается по закону Дюгамеля-Неймана

$$
\sigma_{i j}=E(\mathbf{x})\left(\varepsilon_{i j}-\alpha \theta \delta_{i j}\right),
$$

где $E(\mathbf{x}), \alpha(\mathbf{x}), \theta(\mathbf{x})$ и $\delta_{i j}$ обозначают модуль упругости Юнга и температурный коэффициент линейного расширения неоднородного материала области $\Omega$, разницу между текущей и исходной температурой области и символ Кронекера, соответственно. Поля смещений и температуры связаны через уравнения (1)-(3).

Целью топологической оптимизации является отыскание оптимального распределения материала, которое максимизирует жесткость или минимизирует податливость термоупругой области.

Проблема топологической оптимизации для задачи термоупругости может быть сформулирована как [23]

$$
\min _{0 \leq r(x) \leq 1} C=\iint_{\Omega} \beta \theta u_{i, i} d \Omega+\int_{\Gamma} F u_{3} d \Gamma
$$

при ограничении

$$
\int_{\Omega} \rho(x) d \Omega \leq A(\Omega) \gamma,
$$

где $\beta(\mathbf{x})=\alpha(\mathbf{x}) E(\mathbf{x}), \rho(\mathbf{x})-$ плотность материала; $\gamma-$ коэффициент использования базового материала.

Проектная переменная $r(\mathbf{x})$ связана с модулем Юнга $E(\mathbf{x})$, с $\beta(\mathbf{x})$ и с объемной плотностью материала $\rho(\mathbf{x})$ каждого элемента по следующим соотношениям (схема RAMP) [5]:

$$
\begin{gathered}
E(\mathbf{x})=\frac{E_{0} r(\mathbf{x})}{(1+p \cdot(1-r(\mathbf{x}))}, \\
\beta(\mathbf{x})=\frac{\alpha_{0} E_{0} r(\mathbf{x})}{(1+q \cdot(1-r(\mathbf{x}))}, \\
\rho(x)=\frac{\rho_{0} r(\mathbf{x})}{(1+p \cdot(1-r(\mathbf{x}))}, \mathbf{x} \in \Omega,
\end{gathered}
$$

где $p, q$ - параметры штрафа, используемые для обеспечения компактного распределения материала; $r(\mathbf{x})-$ поле проектных переменных $0<r_{0} \leq r(\mathbf{x}) \leq 1 ; r_{0}-$ малое число, которое гарантирует ненулевую жесткость конечных элементов. При $r(\mathbf{x})=1$ вся область полностью заполнена армирующим базовым материалом. После применения процедуры топологической оптимизации определяются значения модуля упругости $E(\mathbf{x})$, коэффициента $\beta(\mathbf{x})$ и объемной плотности материала $\rho(\mathbf{x})$ для оптимальной топологии микроструктуры.

Полученные значения $E(\mathbf{x}), \beta(\mathbf{x})$ и $\rho(\mathbf{x})$ далее использовались при построении математической модели неоднородной нано балки Тимошенко. 


\section{Математическая модель гибких нанобалок} на основе гипотез Тимошенко

Балка представляет собой область $\Omega$ пространства $R^{2}$, соотнесенную к декартовой системе координат $O X Z$. Срединная линия задана при $z=0$, оси $O X$ и $O Z$ направлены в соответствии с рис. 2. Таким образом, балка определяется как $\Omega=\left\{x \in[0, a] ;-\frac{h}{2} \leq z \leq \frac{h}{2}\right\}$, $0 \leq t \leq \infty$. На основании модифицированной моментной теории [22] запасенная энергия упругой деформации балки $U$ с учетом ее размерно-зависимого поведения может быть записана как

$$
U=\frac{1}{2} \iint_{\Omega}\left(\sigma_{x x} \varepsilon_{x x}+\sigma_{x z} \varepsilon_{x z}+m_{x y} \chi_{x y}\right) d \Omega,
$$

где $\sigma_{x x}, \sigma_{x z}-$ элементы симметричного тензора напряжений $\sigma ; m_{x y}$ - элементы девиаторной части симметричного тензора момента высшего порядка m; $\chi_{x y}$ - элементы тензора кривизны $\chi ; \varepsilon_{x x}, \varepsilon_{x z}$ - элементы симметричного тензора деформаций $\varepsilon$. Система координат, кинематические параметры и нагрузки для балки Тимошенко, смоделированной на основе модифицированной моментной теории, показаны на рис. 2. Нагрузка $f(x, t)$ связана с осевой массовой силой на единицу длины, $C(x, t)$ - распределенные моменты на единицу длины и $q(x, t)$ обозначает интенсивность распределенной поперечной силы на единицу длины. Считается, что свойства балки изменяются как вдоль осевой координаты $O X$, так и в направлении ее толщины, оси $O Z$
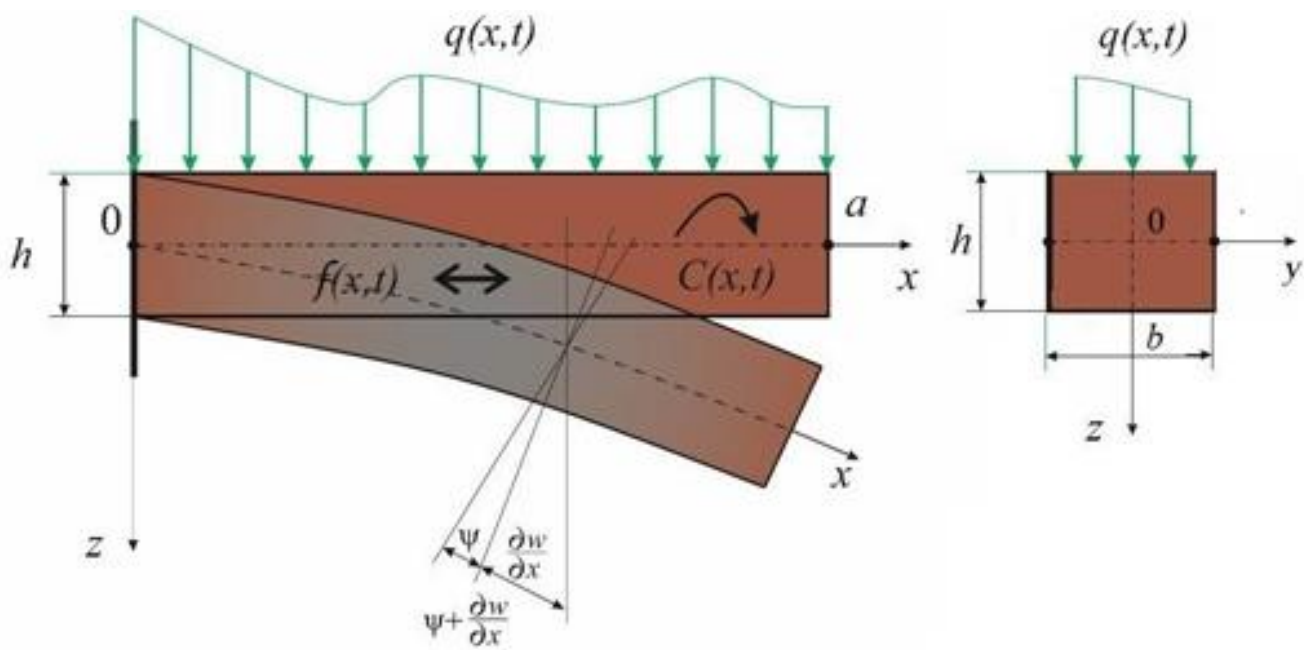

Pис. 2. Расчетная схема балки Тимоменко

Fig. 2. Design model of the Timoshenko beam

Поле смещений в произвольной точка микро балки Тимошенко записываются в виде

$$
u_{1}=u(x, t)-z \psi(x, t), u_{2}=0, u_{3}=w(x, t),
$$

где $u(x, t), w(x, t)$ обозначают осевое смещение и поперечное отклонение точек срединной линии, то есть при $z=0 ; \psi(x, t)$ - угол поворота нормали по отношению к недеформированному состоянию.

Используя нелинейные соотношения Кармана, для модели Тимошенко получим:

$$
\varepsilon_{x x}=u_{, x}+\frac{1}{2}\left(w_{, x}\right)^{2}+z \psi_{, x}, \varepsilon_{x z}=\frac{1}{2}\left(\psi+w_{, x}\right) .
$$

Также из равенства $\phi_{i}=(\operatorname{rot}(\mathbf{u}))_{i} / 2$ можно записать

$$
\phi_{x}=0, \phi_{y}=\frac{1}{2}\left(\psi-w_{, x}\right), \phi_{z}=0 .
$$

Отсюда получаем следующее выражение для единственного неравного нулю компонента симметричной части тензора кривизны:

$$
\chi_{x y}=\chi_{y x}=\frac{1}{4}\left(\psi_{, x}-w_{, x x}\right) .
$$

Определяющие соотношения при наличии температурного поля для неравных нулю компонентов симметричной части тензора напряжения и девиаторной части тензора момента высшего порядка имеют следующие выражения

$$
\begin{gathered}
\sigma_{x x}=E(x, z)\left(\varepsilon_{x x}-\alpha \theta\right), \sigma_{x z}=k_{s} G(x, z)\left(w_{, x}+\psi\right), \\
m_{x y}=2 l^{2} G(x, z) \chi_{x y},
\end{gathered}
$$

где $E(x, z)$ модуль Юнга оптимального распределения материала, полученный на этапе топологической оптимизации балки; $G(x, z)=E(x, z) / 2(1+v)$ - модуль сдвига; $v$ - коэффициент Пуассона, который принят постоянным; $l^{2}$ - материальный параметр длины.

Из уравнений (7)-(12) записана энергия деформации $U$ неоднородной микро балки как

$$
\begin{gathered}
U=\frac{1}{2} \int_{0}^{a} \int_{A}\left(\sigma_{x x} \varepsilon_{x x}+\sigma_{x z} \varepsilon_{x z}+2 m_{x y} \chi_{x y}\right) d A d x= \\
=\frac{1}{2} \int_{0}^{a} \int_{A}\left\{\begin{array}{l}
E\left(u_{, x}+\frac{1}{2}\left(w_{, x}\right)^{2}+z \psi_{, x}\right)^{2}+ \\
+k_{s} G\left(\psi+w_{, x}\right)^{2}+ \\
+\frac{1}{8} 2 l^{2} G\left(\psi_{, x}-w_{, x x}\right)^{2}
\end{array}\right\} d A d x,
\end{gathered}
$$


где $A=h b$ - площадь поперечного сечения балки. Кинетическая энергия балки $K$ определяется выражением:

$$
\begin{gathered}
K=\frac{1}{2} \int_{0}^{a} \int_{A} \rho\left\{\left(u_{, t}-z \psi_{, t}\right)^{2}+w_{, t}^{2}\right\} d A d x= \\
=\frac{1}{2} \int_{0}^{a}\left\{m_{0}\left(u_{, t}\right)^{2}+2 Q u_{, t} \psi_{, t}+\tilde{I}\left(\psi_{, t}\right)^{2}+m_{0}\left(w_{, t}\right)^{2}\right\} d x .(14)
\end{gathered}
$$

Внешняя работа, связанная с распределенными силами, распределенными моментами и усилиями на концах балки имеет вид

$$
\begin{aligned}
W= & \int_{0}^{a}\left(G(x, t) u+q(x, t) w+C(x, t) \theta_{2}\right) d x+ \\
& +\left.\left(\tilde{N} u+\tilde{V} w+\tilde{M}_{\sigma} \psi+\tilde{M}_{M} w_{, x}\right)\right|_{x=0} ^{x=a},
\end{aligned}
$$

где $\tilde{N}$ является осевой результирующей силой нормальных усилий $\sigma_{11}+N_{0} / A ; \tilde{V}$ обозначает поперечную результирующую поперечных усилий; $M_{\sigma} \tilde{M}_{\sigma}-$ внешний изгибающий момент, вызываемый нормальными напряжениями $\sigma_{11} ; M_{M} \tilde{M}_{M}-$ внешний изгибающий момент, вызываемый моментом $m_{12}$.

Внешняя работа, связанная с диссипацией энергии, имеет вид

$$
W_{\varepsilon}=\varepsilon \int_{0}^{a}\left(\frac{\partial u}{\partial t} u+\frac{\partial w}{\partial t} w+\frac{\partial \psi}{\partial t} \psi\right) d x,
$$

где $\varepsilon$ - коэффициент диссипации.

Используем принцип Гамильтона

$$
\delta \int_{t_{1}}^{t_{2}}(K-U+W) d t=0 .
$$

Подставим в (17) выражения (13)-(16) проварьируем по переменным $u, w$ и $\psi$, интегрируем по частям и, наконец, приравнивая выражения перед $\delta u, \delta w$ и $\delta \psi$ к нулю, мы получаем разрешающие уравнения движения, которые представляют собой систему нелинейных дифференциальных уравнений 8-го порядка в частных производных:

$$
\begin{gathered}
{\left[k_{1}\left(u_{, x}+\frac{1}{2}\left(w_{, x}\right)^{2}\right)\right]_{, x}+G=m_{0} u_{, t t}+Q \psi_{, t t},} \\
{\left[k_{2} \psi_{, x}+k_{4}\left(\psi_{, x}-w_{, x x}\right)\right]_{, x}-k_{3}\left(\psi+w_{, x}\right)+\frac{C}{2}=} \\
=Q u_{, t t}+\tilde{I} \psi_{, t t}, \\
\left\{\left[k_{1}\left(u_{, x}+\frac{1}{2}\left(w_{, x}\right)^{2}\right)\right]_{, x}+k_{3}\left(\psi+w_{, x}\right)\right\}_{, x}+ \\
+\left[k_{4}\left(\psi_{, x}-w_{, x x}\right)\right]_{, x x}-q+\frac{C_{, x}}{2}=m_{0} w_{t t},
\end{gathered}
$$

а также граничные условия:

$$
\left.\left[k_{1}\left(u_{, x}+\frac{1}{2}\left(w_{, x}\right)^{2}\right)-\tilde{N}\right]\right|_{x=0, a}=0 \text { или }\left.\delta u\right|_{x=0, a}=0,
$$

$$
\begin{aligned}
& {\left.\left[k_{2} \psi_{, x}+k_{4}\left(\psi_{, x}-w_{, x x}\right)-\tilde{M}_{\sigma}-\frac{\tilde{M}_{m}}{2}\right]\right|_{x=0, a}=0} \\
& \text { или }\left.\delta \psi\right|_{x=0, a}=0 \text {, } \\
& \left.\left\{\begin{array}{l}
\left.\left[k_{1}\left(u_{, x}+\frac{1}{2}\left(w_{, x}\right)^{2}\right)\right] w_{, x}+k_{3}\left(\psi+w_{, x}\right)+\right] \\
+k_{4}\left(\psi_{, x x}-w_{, x x x}\right)+\frac{c}{2}-\tilde{V}
\end{array}\right\}\right|_{x=0, a}=0 \\
& \text { или }\left.\delta w\right|_{x=0, a}=0 \text {, } \\
& \left.\left(k_{4}\left(\psi_{, x}-w_{, x x}\right)+\frac{\tilde{M}_{m}}{2}\right)\right|_{x=0, a}=0 \text { или }\left.\delta w_{, x}\right|_{x=0, a}=0 \text {, }
\end{aligned}
$$

где

$$
\begin{gathered}
k_{1}(\mathrm{x})=\int_{A} E(x, z) d A, k_{2}(x)=\int_{A} E(x, z) z^{2} d A, \\
k_{3}(x)=k_{s} \int_{A} G(x, z) d A, k_{4}(x)=\frac{1}{4} \int_{A} G(x, z) l^{2} d A, \\
m_{0}(x)=\int_{A} \rho(x, z) d A, Q(x)= \\
=\int_{A} \rho(x, z) z d A, \tilde{I}(x)=\int_{A} \rho(x, z) z^{2} d A .
\end{gathered}
$$

Начальные условия имеют вид:

$$
\begin{aligned}
& w(x, 0)=\phi_{1}(x) ; w(x, 0),{ }_{t}=\phi_{2}(x) ; \\
& u(x, 0)=\phi_{3}(x) ; u(x, 0),{ }_{t}=\phi_{4}(x) ; \\
& \psi(x, 0)=\phi_{5}(x) ; \psi(x, 0),{ }_{t}=\phi_{6}(x) .
\end{aligned}
$$

\section{Методы получения численного решения}

На первом этапе при оптимизации топологии микроструктуры, задача (4)-(6), плоской области используется метод конечных элементов. Для этого область $\Omega$ разбивается на конечные элементы, и плотность материала $\rho(\mathbf{x})$ считается постоянной на каждом элементе. Оптимальная топология микроструктуры получена на основе решения плоской задачи термоупругости с использованием метода подвижных асимптот [24]. После получения оптимальных значений массивы значений модуля упругости, плотности (6) подставляются в нелинейные дифференциальные уравнения в частных производных математической модели нано балки Тимошенко.

Уравнения в частных производных с переменными коэффициентами, граничные и начальные условия (19)-(21) методом конечных разностей, с аппроксимацией $O\left(h^{2}\right)$ по пространственным переменным, приведены к задаче Коши. В свою очередь задача Коши решается методом Рунге-Кутта 4 порядка. Подбор оптимального шага в численных методов проводился с помощью принципа Рунге.

Результаты решения задач статики были получены с помощью динамических уравнений (19). Такой подход носит название метода установления или метода «тяжелого шарика». Метод установления имеет широкий диапазон применения. Этот подход позволяет решить нелинейные уравнения различного типа 
(дифференциальные, алгебраические и др.) за счет линеаризации исходной задачи. Это происходит из-за добавления дифференциального оператора по времени. Метод обладает высокой точностью со скоростью сходимости. Кроме этого, если исходная стационарная задача имеет дифференциальный оператор высокого порядка, то за счет второй производной по времени происходит понижение порядка исходной задачи.

В случае если нагрузка $q$ не зависит от времени, мы имеем возможность получить решение статической задачи на основе динамического подхода. Начальное условие играет роль возмущения в статической задаче, а член с первой производной по времени, содержащий коэффициент диссипации, является силой затухания возмущенного решения. Решение динамической задачи можно проводить любым методом решения задачи Коши. В процессе установления возмущенного решения мы приходим к решению статической задачи.

\section{Пример использования методологии} топологической оптимизации

Исследования проводились для двумерной области, размеры $a=40 \mathrm{HM}, h=40 \mathrm{Hм}$, материал - сталь $\left(E_{0}=200 \mathrm{MPa}, \alpha_{0}=12,3 * \mathrm{e}-61 / \mathrm{K}, \rho_{0}=7800 \mathrm{\kappa} \Gamma \mathrm{m}^{3}, v=0,3\right)$. Нагрузка, сосредоточенная в центре, т. е. граница $\Gamma_{3}$, представляет собой одну точку ( $x=20$ нм), граничные условия:

$$
\left.u\right|_{x=0, a}=0,\left.\quad \psi\right|_{x=0, a}=0,\left.w\right|_{x=0, a}=0,\left.w_{, x}\right|_{x=0, a}=0,
$$

начальные условия:

$$
\begin{gathered}
w(x, 0)=0, w(x, 0),_{t}=0, u(x, 0)=0, \\
u(x, 0),_{t}=0, \psi(x, 0)=0, \psi(x, 0),{ }_{t}=0 .
\end{gathered}
$$

Для численного исследования также приняты следующие упрощения: $f(x, t)=0, C(x, t)=0$. Численные результаты получены для задачи в безразмерных переменных. Безразмерные переменные вводились по формулам, приведенным в [25].

В табл. 1 приведена оптимальная топология распределения материала для нано балок, при действии температур $\theta= \pm 100,0$. Здесь красный цвет обозначает армирующий базовый материал.

Используя полученную оптимальную микроструктуру балок, по соотношениям (20) были вычислены значения $k_{1}(x), k_{2}(x), k_{3}(x)$, которые являются переменными по длине балки. Графики этих величин приведены в табл. 2.

Полученные значения были использованы для исследования статических и динамических задач нелинейной балки модели Тимошенко.

Таблица 1. Микроструктуры оптимальных балок

Table 1. Microstructures of optimal beams

$\theta=0$ (100

Таблица 2. Зависимости $k_{1}, k_{2}, k_{3}$ по длине балки

Table 2. Dependences $k_{1}, k_{2}, k_{3}$ on the beam length

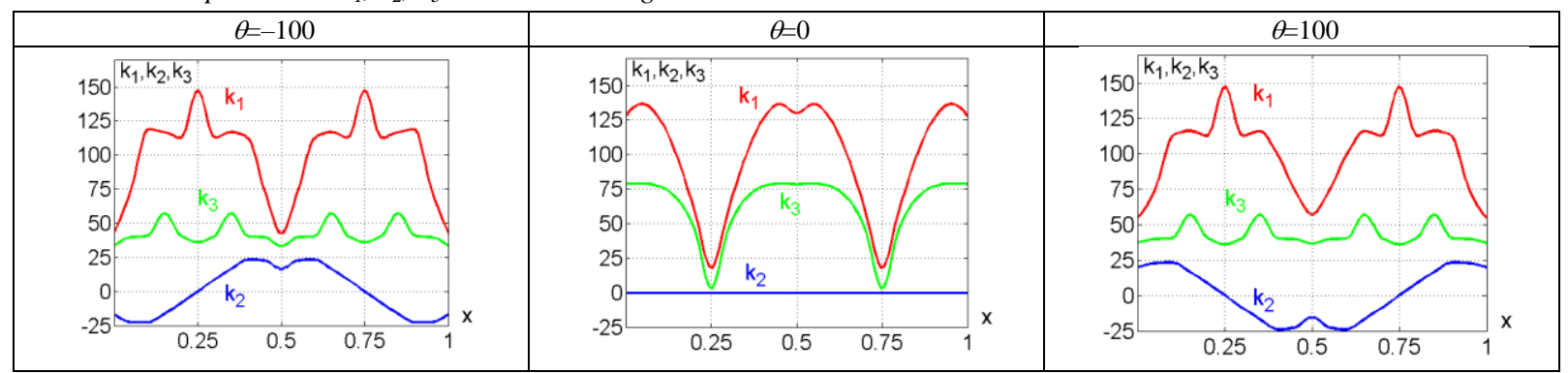

\section{Результаты решения статических задач}

С помощью описанных выше методов было проведено исследование для четырех различных комбинаций параметров: 1 - без учета размерно-зависимого эффекта и однородная; 1 * - без учета размерно-зависимого эффекта, но с учетом оптимизации; 2 - с учетом размернозависимого эффекта, однородная; $2 *$ - с учетом размерно-зависимого эффекта, оптимальная.

Выигрыш, т. е. уменьшение максимального прогиба топологически оптимальной балки по сравнению с однородной, для $\theta=-100$ составляет при q=500 12,5\% для задачи без учета размерно-зависимого поведения и $10,1 \%$ для задачи с учетом размерно-зависимого поведения. Для $\theta=+100$ выигрыш составляет при $q=50012,3 \%$ для задачи без учета размернозависимого поведения и 10,5 \% для задачи с учетом размерно-зависимого поведения. При отсутствии теплового поля выигрыши составляют 13,4 и 11,1 \% соответственно. На рис. 3 приведен график нагрузка прогиб $q(w)$ для нелинейной задачи при $\theta=0$. Анализ результатов показал, что для используемых значений коэффициентов и результатов оптимизации графики $q(w)$ для оптимальной и неоптимальной задач как без учета размерно-зависимого поведения, так и с учетом 
размерно-зависимого поведения существенно отличны при больших прогибах.

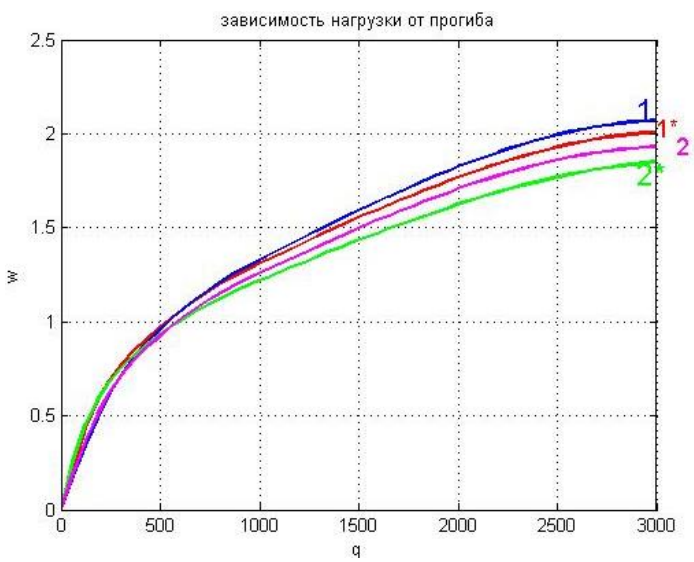

Pис. 3. Зависимость нагрузка-прогиб $q($ w) для $\theta=0$

Fig. 3. Load-deflection dependence $q(w)$ for $\theta=0$

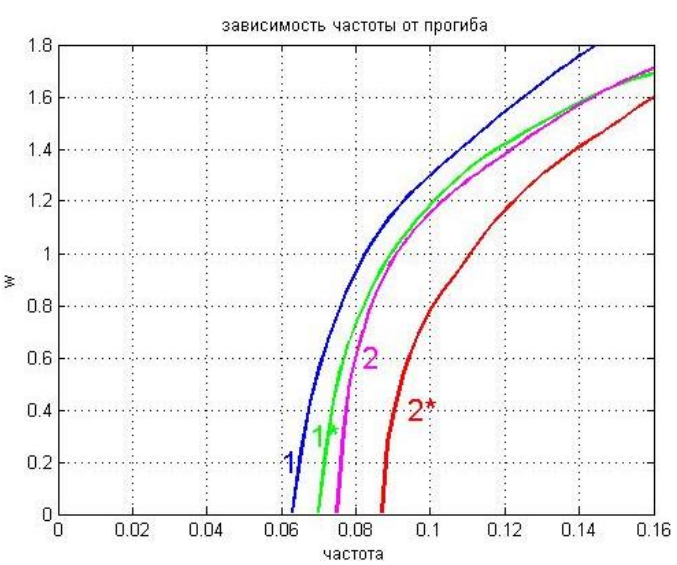

Puc. 4. График частота-прогиб $\omega($ w) для $\theta=0$

Fig. 4. Frequency-deflection graph $\omega(w)$ for $\theta=0$

Как и для значений прогиба имеется выигрыш от оптимизации для значений собственных частот для размерно-зависимой задачи и для задачи без учета размерно-зависимого поведения. Для температуры $\theta=100$ разница между значениями частот для однородной и неоднородной (оптимальной) балок, при значении прогиба 0,00001, без учета размернозависимого поведения составляет 8,7 \%. Для балок с учетом размерно-зависимого поведения 11,76 \%. Для прогиба, равного 1, без учета размерно-зависимого поведения разница составляет 9,9\%, 12,15 \% - с учетом размерно-зависимого поведения.

Для температуры $\theta=-100$ при прогибе 0,00001 разница между результатами оптимальной и неоптимальной балок составляет 7,4 и 5,1 \% соответственно и для прогиба, равного 1, разница составляет 4,4 и $4,47 \%$. При $\theta=0$ для прогиба 0,00001 разница между результатами оптимальной и неоптимальной балок составляет 10,5 и 13,1 \%, для прогиба, равного 1, ра3ница равна 11,4 и 19,2 \%. Таким образом, зависимость частота-прогиб $\omega(w)$ для всех случаев $(\theta=-100$, $\theta=0, \theta=100)$ демонстрируют отличие результатов для оптимальной и однородной балок.
На основании полученных результатов можно сделать вывод, что разработанный авторами алгоритм и программа оптимизации позволяют получать конструкции с оптимальными свойствами как с учетом размерно-зависимого поведения, так и без учета размерно-зависимого поведения.

\section{Исследование нелинейной динамики}

Для исследования влияния оптимальной топологии балки на нелинейную динамику использовались основные характеристики: спектр Фурье, вейвлет спектр, построенный на базе материнского вейвлета Морле, сечение Пуанкаре, фазовый портрет и значение старшего показателя Ляпунова. Результаты получены при действии знакопеременной поперечной нагрузки $q=q_{0} \sin \left(\omega_{p} t\right)$. Коэффициент диссипации взят в виде $\varepsilon=1$. Частота возбуждения $\omega_{p}=9$, амплитуда внешней нагрузки $q_{0}=14000$. Исследование старших показателей Ляпунова - скорости расходимости траекторий в фазовом пространстве, проводились для выяснения степени хаотизации колебаний. Если старший показатель Ляпунова положителен, колебания хаотические, при стремлении его к нулю колебания становятся квазигармоническими, отрицательный показатель Ляпунова - гармонические колебания. Для проверки достоверности получаемых значений старших показателей Ляпунова использовались три метода, основанные на разных алгоритмах. Метод Wolf [25] рассчитывает старший показатель Ляпунова на основе слежения за долгосрочными темпами роста элементов малого объема в аттракторе. Метод Rosenstein [26] показатель Ляпунова вычисляет как угол наклона наиболее линейного участка функции разностей расстояний между траекториями. Алгоритм Kantz [27] рассчитывает старший показатель Ляпунова путём поиска всех соседей в окрестности опорной траектории и высчитывает среднюю дистанцию между соседями и опорной траекторией как функцию от времени (или относительное время, умноженное на частоту выборки данных).

В табл. 3 приведены результаты нелинейной динамики для нано балок. Без учета размернозависимого поведения $(\ln =0)$ : неоптимальная балка $(\mathrm{Opt}=0)$ (случай 1) и оптимальная балка $(\mathrm{Opt}=1)($ случай 2), а также с учетом размерно-зависимого поведения $(\mathrm{Ln}=0,3)$ : неоптимальная балка $(\mathrm{Opt}=0)$ (случай 3) и оптимальная балка $(\mathrm{Opt}=1)$ (случай 4). Рассмотрены следующие характеристики: а) сигнал $w(0,5 ; t)$; б) спектр Фурье, построенный на основании быстрого преобразования Фурье $S(w)$; в) 2D вейвлет-спектр $\dot{w}[w(t)]$.

Для случая 1 ( $\ln =0$, неоптимальная) спектр частот (1б) содержит семь основных частот $\omega_{1}=0,91$, $\omega_{2}=1,12, \omega_{3}=2,17, \omega_{4}=3,47, \omega_{5}=3,71, \omega_{6}=4,76, \omega_{7}=7,96$. Между частотами имеются следующие соотношения: $\omega_{p}-\omega_{7}=\omega_{6}-\omega_{5}=\omega_{3}-\omega_{2}=1,04, \quad \omega_{2}-\omega_{1}=\omega_{5}-\omega_{4}=0,23$, $\omega_{7}-\omega_{6}=2\left(\omega_{4}+\omega_{3}\right)$. Таким образом, все частоты зависимы.

Для случая 2 ( $\ln =0$, оптимальная) спектр Фурье содержит следующие основные частоты $\omega_{1}=0,26, \omega_{2}=4,24$. Связь между частотами $\omega_{p}=2\left(\omega_{1}+\omega_{2}\right)$. 
Таблица 3. Результаты для динамической задачи

Table 3. Results for a dynamic problem

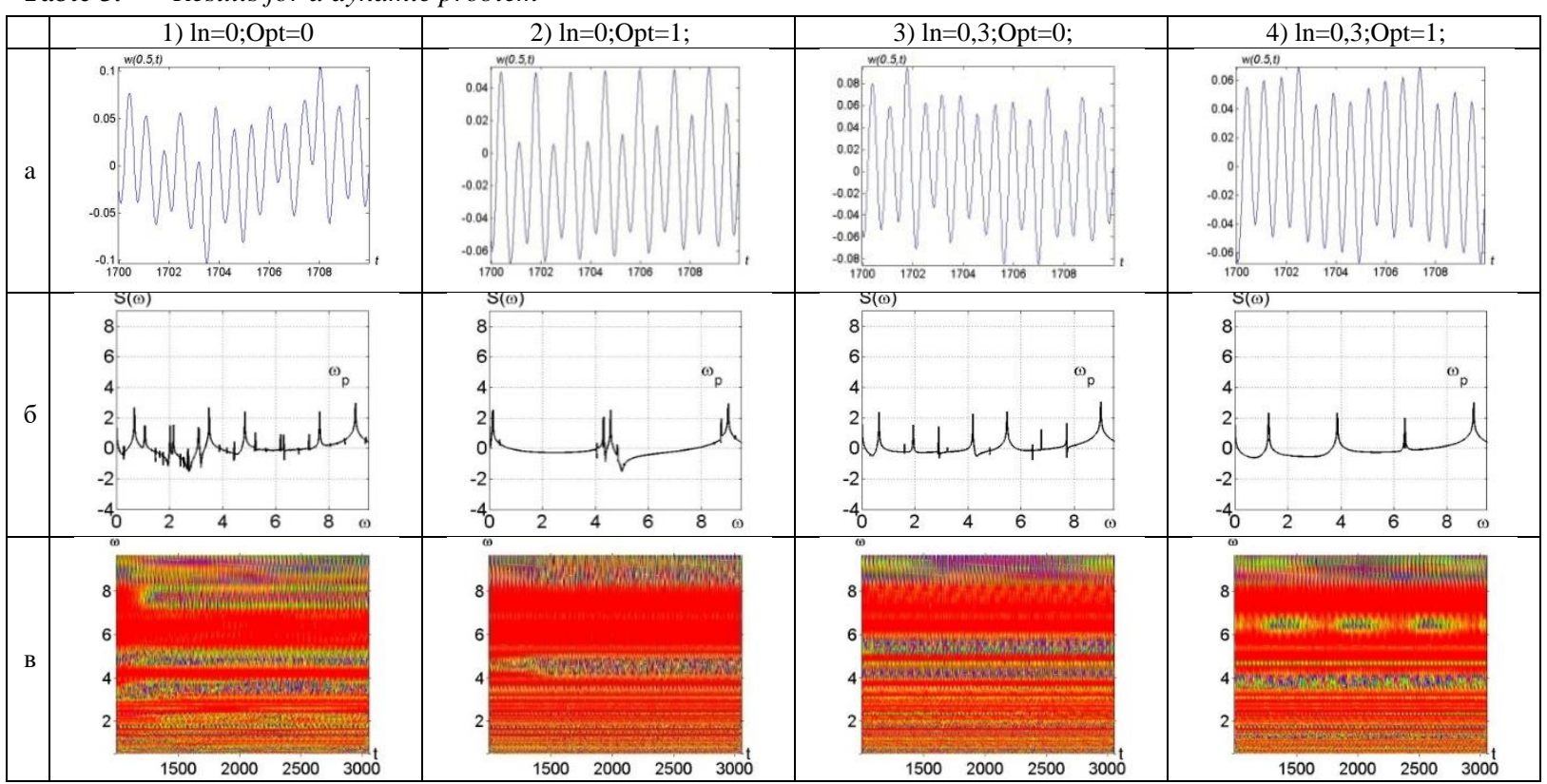

Для случая $3(\ln =0$, неоптимальная) спектр частот (2b) содержит шесть основных частот с $\omega_{1}=0,42$, $\omega_{2}=2,05, \omega_{3}=2,62, \omega_{4}=4,1, \omega_{5}=5,21, \omega_{6}=7,87$. Между этими частотами выполняется соотношение $\omega_{p}=\omega_{7}+\omega_{2}$, $\omega_{p}=\omega_{4}+\omega_{3}+\omega_{2}+\omega_{1}$.

Для случая $4(\ln =0,3$, оптимальная) спектр частот (2б) содержит три основные частоты $\omega_{1}=1,32, \omega_{2}=3,91$, $\omega_{3}=6,44$. Между этими частотами выполняется соотношение $\omega_{2}-\omega_{1}=\omega_{3}-\omega_{2}=2,52$.

Сравнение результатов однородной и оптимальной балок показывает, что динамические характеристики существенно отличаются, как для балок с учетом нано размера, так и без его учета. При этом частотный характер колебаний существенно меняется, что отчетливо видно на графиках Фурье и вейвлет спектров - количество частот существенно уменьшается. Старшие показатели Ляпунова, вычисленные по трем методам: Wolf, Kantza и Rosenstein, показывают, что для всех четырех случаев присутствует хаотизация колебаний - старший показатель Ляпунова положителен. Однако для оптимальных балок старшие показатели Ляпунова по трем методам близки к нулю, что говорит о стремлении системы к гармоническим колебаниям. В случае неоптимальных балок старшие показатели Ляпунова положительны и существенно отличны от нуля.

Таким образом, построение оптимально микроструктуры нано балки позволяет изменить динамические режимы при заданных граничных условиях и типе нагрузки. В свою очередь это позволит продлить срок службы элементов НЭМС.

\section{Заключение}

Проведен сравнительный анализ нелинейной динамики однородных балок и неоднородных балок с оптимальным распределением материала по толщине и длине. Построена методология получения оптимальной микроструктуры нано балки на основе топологической оптимизации по критерию минимума податливости (максимума жесткости). Построена математическая модель балки на основе кинематических гипотез Тимошенко и на базе модифицированной моментной теории, с учетом геометрической нелинейности по Карману и воздействия теплового поля по закону Дюамеля-Неймана. Модель описывается системой нелинейных дифференциальных уравнений в частных производных с переменными коэффициентами. Переменные коэффициенты используются при моделировании балки с оптимальной топологией. В случае однородной (неоптимальной) балки коэффициенты постоянны по величине.

Проведено сравнение статических и динамических результатов оптимальных и однородных балок для различных значений масштабного параметра длины материала и температуры. Численное исследование показало, что прогиб балки с оптимальной микроструктурой меньше, чем для неоптимальной (однородной), причем выигрыш составляет около $12 \%$ для полноразмерных балок и около 10 \% для нано балок. Собственные частоты колебаний оптимальных балок отличаются от неоптимальных в среднем на 16 и $13 \%$ соответственно. Таким образом, при использовании топологической оптимизации был создана балка с новыми улучшенными свойствами. Применение этого алгоритма при создании элементов нано-электромеханических систем для нефтегазодобывающей техники позволит создавать приборы с большим срокам службы, приспособленные к более тяжелым условиям эксплуатации.

Работа выполнена при финансовой поддержке гранта РНФ № 16-11-10138-П. 


\section{СПИСОК ЛИТЕРАТУРЬ}

1. Кишов Е.А., Комаров В.А. Топологическая оптимизация силовых конструкций методом выпуклой линеаризации // Вестник Самарского университета. Аэрокосмическая техника, технологии и машиностроение. - 2018. - Т. 17. - № 1. - С. 137-149.

2. Товстик П.Е., Товстик Т.П. Уравнения равновесия сильно неоднородной по толщине пологой оболочки // Доклады Академии наук. - 2017. - Т. 477. - № 3. - С. 295-299.

3. Ватульян А.О., Плотников Д.К., Поддубный А.А. О некоторых моделях индентирования функционально градиентных покрытий // Известия Саратовского университета. Новая серия. Серия: Математика. Механика. Информатика. - 2018. T. 18. - № 4. - C. 421-432

4. Моисеенко И.А. Распространение нормальных волн вдоль трансверсально изотропных функционально градиентных цилиндров // Вестник Донецкого национального университета. Серия А: Естественные науки. - 2018. - № 1. - С. 37-54.

5. Barroqueiro B., Andrade-Campos A., Valente R.A.F. Designing self supported SLM structures via topology optimization // Journal of Manufacturing and Materials Processing. - 2019. - V. 3 (3) URL: https://doi.org/10.3390/jmmp3030068 (дата обращения 12.02.2020).

6. Павлов С.П., Бодягина К.С., Крысько В.А. К вопросу об оптимизации формы геологических выработок и топологии их укрепления // Известия Томского политехнического университета. Инжиниринг георесурсов. - 2017. - Т. 328. - № 1. C. 6-13.

7. Xie Y., Steven G. A simple evolutionary procedure for structural optimization // Computers and Structures. - 1993. - № 49. P. 885-896.

8. Allaire G., Jouve F., Toader A. Structural optimization using sensitivity analysis and a level-set method // Journal of Computational Physics. - 2004. - № 194. - P. 363-393.

9. Li Q., Steven G., Xie Y. Thermoelastic topology optimization for problems with varying temperature fields // Journal of Thermal Stresses. - 2001 - № 24 - P. 347-366.

10. Rodrigues H., Fernandes P. A material based model for topology optimization of thermoelastic structures // International Journal of Numerical Methods in Engineering. - 1995. - № 38 (12). P. 1951-1965.

11. Cho S., Choi J. Efficient topology optimization of thermoelasticity problems using coupled field adjoint sensitivity analysis method // Finite Elements in Analysis and Design. - 2005. № 41 (15). - P. 481-495.

12. Короленко В.А. Решение задачи об изгибе балки с трещиной в рамках градиентной теории упругости // Гагаринские чтения2019. Сборник тезисов докладов XLV Международной молодежной научной конференции. - М., 2019. - С. 961-962.

13. Altenbach H., Eremeyev V.A. Strain rate tensors and constitutive equations of inelastic micropolar materials // International Journal of Plasticity. -2014. - № 63. - P. 3-17.

14. Chen Y., Lee J D. Determining material constants in micromorphic theory through phonon dispersion relations // International Journal Engineering Science. - 2003. - № 41. P. 871-886.
15. Micromorphic prism element / R. Ansari, M. Bazdid-Vahdati, A. Shakouri, A. Norouzzadeh, H. Rouhi // Mathematics and Mechanics of Solids. - 2016. - DOI: 10.1177/ 1081286516637115

16. Фу Ч., Ян С. Анализ изгиба балки Тимошенко с трещиной с использованием нелокальной градиентной теории упругости // Прикладная механика и техническая физика. - 2019. - Т. 60. № 3 (355). - C. 196-206.

17. Васильев В.В., Лурье С.А. О корректных нелокальных обобщенных теориях упругости // Физическая мезомеханика. 2016. - Т. 19. - № 1. - С. 47-59.

18. Кувыркин Г.Н., Савельева И.Ю. Термомеханическая модель нелокального деформирования твердого тела // Известия Российской академии наук. Механика твердого тела. - 2016. № 3. - C. 20-27.

19. Couple stress based strain gradient theory for elasticity / F. Yang, A.C.M. Chong, D.C.C. Lam, P. Tong // International Journal of Solids and Structures. - 2002. - № 39. - P. 2731-2743.

20. Барулина М.А., Папкова И.В., Крысько А.В. Динамика круглого чувствительного элемента наноэлектромеханического датчика // Юбилейная XXV Санкт-Петербургская Международная конференция по интегрированным навигационным системам. Сборник материалов / Гл. ред. В.Г. Пешехонов. СПб., 2018. - С. 216-219.

21. Тимошенко С.П., Войновский-Кригер С. Пластины и оболочки. - М.: Наука, 1966. - 636 с.

22. Павлов С.П., Жигалов М.В. Новая размерно-зависимая модель для нелинейных конструктивно неоднородных балок Тимошенко // Математические методы в технике и технологиях MMTT. - 2015. - № 1 (71). - C. 92-95.

23. Оптимизация армирования элементов микромеханических приборов для геологических изысканий / С.П. Павлов, Н.В. Бекренев, И.В. Злобина, К.С. Бодягина // Известия Томского политехнического университета. Инжиниринг георесурсов. - 2018. - Т. 329. - № 3. - С. 44-52.

24. Колесников В.Л., Бракович А.И. Вероятностные модели с подвижными асимптотами для оптимизации систем с последовательно-параллельным соединением элементов // Труды БГТУ. Серия 3: Физико-математические науки и информатика. - 2018. - № 2 (212). - C. 101-106.

25. Nonlinear behaviour of different flexible size-dependent beams models based on the modified couple stress theory. Part 2. Chaotic dynamics of flexible beams / A.V. Krysko, J. Awrejcewicz, M.V. Zhigalov, S.P. Pavlov, V.A. Krysko // International Journal of Non-Linear Mechanics. - 2017. - № 93. - P. 106-121.

26. Крысько В.А. мл., Мицкевич С.А. Хаотические колебания размернозависимой гибкой нанооболочки // Компьютерные науки и информационные технологии Материалы Международной научной конференции. - Саратов: ИЦ «Наука», 2018. C. 205-208.

27. Хаотические колебания геометрически нелинейных наноразмерных пологих осесимметричных оболочек / В.А. Крысько мл., А.В. Кириченко, И.В. Папкова, И.Е. Кутепов // Проблемы прочности и пластичности. - 2018. - Т. 80. - № 4. - С. 446-455.

Поступила 16.06.2020 2.

\section{Информация об авторах}

Павлов С.П., доктор физико-математических наук, профессор кафедры математики и моделирования, Саратовский государственный технический университет им. Гагарина Ю.А.

Жигалов М.В., доктор физико-математических наук, профессор кафедры математики и моделирования, Саратовский государственный технический университет им. Гагарина Ю.А.

Захарова $\boldsymbol{A}$.A., доктор технических наук, профессор кафедры информатики и программного обеспечения Брянского государственного технического университета.

Крысько В.А., доктор технических наук, профессор, заведующий кафедрой математики и моделирования, Саратовский государственный технический университет им. Гагарина Ю.А. 
UDC 539.3

\title{
NONLINEAR DYNAMICS OF TOPOLOGICALLY OPTIMAL TIMOSHENKO NANOBEAM BASED ON THE COUPLE STRESS THEORY
}

\author{
Sergey P. Pavlov 1 \\ pspsar@yandex.ru
}

\author{
Maksim V. Zhigalov ${ }^{1}$, \\ zhigalovm@yandex.ru \\ Alena A. Zakharova², \\ zaa@tu-bryansk.ru \\ Vadim A. Krysko', \\ tak@san.ru \\ 1 Yuri Gagarin Saratov State Technical University, \\ 77, Politekhnicheskaya street, Saratov, 410054, Russia. \\ 2 Bryansk State Technical University, \\ 7, blvd. 50 let Octyabrya, Bryansk, 241035, Russia.
}

The relevance of research. Technological sensors (sensors of load, pressure, liquid temperature, solution yield, liquid density) are used for monitoring data of technical operations of well drilling, various types repair of well, exploration drilling in the oil and gas industry on drilling and repair of all types of wells. Modern sensors are small in size and manufactured on the basis of nanoelectromechanical systems to increase sensitivity. They include constituent elements, nano beams and nano plates. These elements operate at high temperatures and are subjected to mechanical loads of various kinds. Increasing the strength of these elements is undoubtedly an urgent task.

The main aim of the research is to build a mathematical model on the basis of Timoshenko kinematic hypothesis and the modified couple stress theory of a sensing element in the form of a beam, a nano-electro-mechanical sensor under the action of mechanical and thermal fields; to develop methodology for obtaining the optimal topology of nano beams for arbitrary static and dynamic loads and different boundary conditions in order to increase its rigidity; to conduct a comparative analysis of the statics and nonlinear dynamics of optimal and nonoptimal beams.

Objects: element of nanoelectromechanical systems in the form of a beam taking into account the optimal microstructure.

Methods: methods of topological optimization, variational methods, second order finite difference method, Runge-Kutta type methods, Fourier and wavelet analysis, phase portrait and Poincare section.

Results. A methodology for obtaining the optimal microstructure of the nano beam based on topological optimization is developed. On the basis of the Hamilton-Ostrogradsky principle, a mathematical model of a Timoshenko nano beam inhomogeneous in two directions (in thickness and length) is constructed on the basis of a modified moment theory. A comparative analysis of static bending and nonlinear dynamics was performed for optimal and non-optimal nano beams.

\section{Key words:}

Timoshenko nanobeam, nanoelectromechanical systems, temperature and force fields, modified couple stress theory of elasticity, finite difference method, Runge-Kutta type methods, topological optimization, nonlinear dynamics.

The research was financially supported by the grant RNF no. 16-11-10138-П.

\section{REFERENCES}

1. Kishov E.A., Komarov V.A. Topological optimization of power structures by convex linearization. Vestnik of Samara University. Aerospace and Mechanical Engineering, 2018, vol. 17, no. 1, pp. 137-149. In Rus.

2. Tovstik P.E., Tovstik T.P. The equation of equilibrium of strongly non-uniform thickness shellsю Doklady Akademii nauk, 2017, vol. 477, no. 3, pp. 295-299. In Rus.

3. Vatulyan A.O., Plotnikov D.K., Poddubny A.A. On some models of functional gradient coatings indentation. Izvestiya of Saratov University. New Series. Series: Mathematics. Mechanics. Informatics, 2018, vol. 18, no. 4, pp. 421-432. In Rus.

4. Moiseenko I.A. Propagation of normal waves along a transversely isotropic functionally graded cylinders. Vestnik Doneckogo nacional'nogo universiteta. Seriya A: Estestvennye nauki, 2018, no. 1, pp. 37-54. In Rus.

5. Barroqueiro B., Andrade-Campos A., Valente R.A.F. Designing self supported SLM structures via topology optimization. Journal of Manufacturing and Materials Processing, 2019, no. 3 (3). Available at: https://doi.org/10.3390/jmmp3030068 (accessed 12 February 2020).
6. Pavlov S.P., Bodyagina K.S., Krysko V.A. To the question of optimization of the form of geological workings and topology of their strengthening]. Bulletin of the Tomsk Polytechnic University. Geo Assets Engineering, 2017, vol. 328, no. 1, pp. 6-13. In Rus.

7. Xie Y., Steven G. A simple evolutionary procedure for structural optimization. Computers and Structures, 1993, no. 49, pp. 885-896.

8. Allaire G., Jouve F., Toader A. Structural optimization using sensitivity analysis and a level-set method. Journal of Computational Physics, 2004, no. 194, pp. 363-393.

9. Li Q., Steven G., Xie Y. Thermoelastic topology optimization for problems with varying temperature fields. Journal of Thermal Stresses, 2001, no. 24, pp. 347-366.

10. Rodrigues H., Fernandes P. A material based model for topology optimization of thermoelastic structures. International Journal of Numerical Methods in Engineering, 1995, no. 38 (12), pp. 1951-1965.

11. Cho S., Choi J. Efficient topology optimization of thermoelasticity problems using coupled field adjoin sensitivity analysis method. Finite Elements in Analysis and Design, 2005, no. 41 (15), pp. 1481-1495.

12. Korolenko V.A. Reshenie zadachi ob izgibe balki s treshchinoy v ramkakh gradientnoy teorii uprugosti [Solution of the problem of 
bending a beam with a crack in the framework of the gradient theory of elasticity]. Gagarinskie chteniya-2019. Sbornik tezisov dokladov XLV Mezhdunarodnoy molodezhnoy nauchnoy konferentsii [Gagarin's readings-2019. Collection of abstracts of the XLV International youth scientific conference ]. Moscow, 2019. pp. 961-962.

13. Altenbach H., Eremeyev V.A. Strain rate tensors and constitutive equations of inelastic micropolar materials. International Journal of Plasticity, 2014, no. 63, pp. 3-17.

14. Chen Y., Lee J.D. Determining material constants in micromorphic theory through phonon dispersion relations. International Journal Engineering Science, 2003, no. 41, pp. 871886.

15. Ansari R., Bazdid-Vahdati M., Shakouri A., Norouzzadeh A., Rouhi H. Micromorphic prism element. Mathematics and Mechanics of Solids, 2016. DOI: 10.1177/1081286516637115.

16. Fu Ch., Yan S. Analysis of the bending of a Timoshenko beam with a crack using the nonlocal gradient elasticity theory. Journal of Applied Mechanics and Technical Physics, 2019, vol. 60, no. 3 (355), pp. 196-206. In Rus.

17. Vasil'ev V.V., Lur'e S.A. The correct generalized nonlocal theories of elasticity. Physical Mesomechanics journal, 2016, vol. 19, no. 1, pp. 47-59. In Rus.

18. Kuvyrkin G.N., Savel'eva I.Yu. Thermomechanical model of nonlocal deformation of a solid body. Mechanics of Solids, 2016, no. 3, pp. 20-27. In Rus.

19. Yang F., Chong A.C.M., Lam D.C.C., Tong P. Couple stress based strain gradient theory for elasticity. International Journal of Solids and Structures, 2002, no. 39, pp. 2731-2743.

20. Barulina M.A., Papkova I.V., Krysko A.V. Dinamika kruglogo chuvstvitelnogo elementa nanoelektromekhanicheskogo datchika [Dynamics of the round sensing element of the nanoelectromechanical sensor]. Yubileynaya XXV Sankt-Peterburgskaya Mezhdunarodnaya konferentsiya po integrirovannym navigatsionnym sistemam. Sbornik materialov [Anniversary XXV St. Petersburg international conference on integrated navigation systems. Collection of materials]. St-Petersburg, 2018. pp. 216-219.
21. Timoshenko S.P., Voynovskiy-Kriger S. Plastiny $i$ obolochki [Plates and shells]. Moscow, Nauka Publ., 1966. 636 p.

22. Pavlov S.P., Zhigalov M.V. Novaya razmerno-zavisimaya model dlya nelineynykh konstruktivno neodnorodnykh balok Timoshenko $[\mathrm{A}$ new size-dependent model for nonlinear structurally inhomogeneous Timoshenko beams]. Matematicheskie metody v tekhnike i tekhnologiyah - MMTT, 2015, no. 1 (71), pp. 92-95.

23. Pavlov S.P., Bekrenev N.V., Zlobina I.V., Bodyagina K.S. Optimization of reinforcement of elements of micromechanical devices for geological surveys. Bulletin of the Tomsk Polytechnic University. Geo Assets Engineering, 2018, vol. 329, no. 3, pp. 44-52. In Rus.

24. Kolesnikov V.L., Brakovich A.I. Probabilistic models with movable asymptotes for optimization of systems with seriesparallel connection of elements. Proceedings of BSTU. Physics and Mathematics. Informatics, 2018, no. 2 (212), pp. 101-106. In Rus.

25. Krysko A.V., Awrejcewicz J., Zhigalov M.V., Pavlov S.P., Krysko V.A. Nonlinear behaviour of different flexible sizedependent beams models based on the modified couple stress theory. Part 2. Chaotic dynamics of flexible beams. International Journal of Non-Linear Mechanics, 2017, no. 93, pp. 106-121.

26. Krysko V.A. Jr, Mickevich S.A. Khaoticheskie kolebaniya razmernozavisimoy gibkoy nanoobolochki [Chaotic oscillations of the dimension dependent flexible nanoshell]. Kompyuternye nauki $i$ informatsionnye tekhnologii. Materialy Mezhdunarodnoy nauchnoy konferentsii [Computer science and information technology Materials of the International scientific conference]. Saratov, Nauka Publ., 2018. pp. 205-208.

27. Krys'ko ml. V.A., Kirichenko A.V., Papkova I.V., Kutepov I.E. Chaotic oscillations of geometrically nonlinear nanoscale sloping axisymmetric shells. Problemy prochnosti i plastichnosti, 2018, vol. 80, no. 4, pp. 446-455. In Rus.

Received: 16 June 2020.

\section{Information about the authors}

Sergey P. Pavlov, Dr. Sc., professor, Yuri Gagarin Saratov State Technical University.

Maksim V. Zhigalov, Dr. Sc., professor, Yuri Gagarin Saratov State Technical University.

Alena A. Zakharova, Dr. Sc., professor, Bryansk State Technical University.

Vadim A. Krysko, Dr. Sc., professor, head of the Mathematics and Modeling Department, Yuri Gagarin Saratov State Technical University. 